\title{
Complete Solution of the Five-Position Synthesis for Spherical Four-Bar Mechanisms
}

Chen-Chou Lin

Associate Professor, Department of Mechanical and Marine Engineering, National Taiwan Ocean University, Keelung, Taiwan R.O.C.

Follow this and additional works at: https://jmstt.ntou.edu.tw/journal

Part of the Mechanical Engineering Commons

\section{Recommended Citation}

Lin, Chen-Chou (1998) "Complete Solution of the Five-Position Synthesis for Spherical Four-Bar Mechanisms," Journal of Marine Science and Technology. Vol. 6: Iss. 1, Article 3.

DOI: $10.51400 / 2709-6998.2516$

Available at: https://jmstt.ntou.edu.tw/journal/vol6/iss1/3

This Research Article is brought to you for free and open access by Journal of Marine Science and Technology. It has been accepted for inclusion in Journal of Marine Science and Technology by an authorized editor of Journal of Marine Science and Technology. 


\title{
COMPLETE SOLUTION OF THE FIVE-POSITION SYNTHESIS FOR SPHERICAL FOUR-BAR MECHANISMS
}

\author{
Chen-Chou Lin*
}

Key words: Spherical mechanism, Path generation, Motion generation, Continuation method, Five precision points.

\section{ABSTRACT}

The objective of this paper is to synthesis spherical four-bar motion and path generators using continuation method (homotopy method). Using continuation method, we may obtain a complete set of the solutions to the problems, and provide mechanism designers with all possible choices which meet the kinematic specifications. In contrast to other local numerical schemes such as Newton method and Powell method which are sensitive to initial guess, continuation method is a global convergence method that guarantee to find all solutions to the equations. In this paper, the problems with four and five precision points will be formulated, solved, and compared with the past results. We use the concept of graphical inversion and pole method to derive the design equations. Equations derived from such method are relatively simple. The total degree of the design equations is lower than other methods. Hence we may solve the design problems effectively. A reliable computer code using the numerical scheme has been developed and applied. The resulting mechanisms will be classified by their rotatability, and mechanisms with circuit and order defects will be examined.

\section{INTRODUCTION}

For the problems of mechanism dimensional synthesis, the synthesis of path generators means to design the dimension of the linkage so that a couplerpoint may trace a curve approximating the desired path. In general, there are two ways of approximating a curve. One is to make a point on the linkage pass the selected precision-points on the desired curve. The other way is application of optimization technique such as least-square method to make the total deviation between the traced curve and the desired path minimized. For the first approach, we may

Paper Received January, 1998. Revised April, 1998. Accepted April, 1998. Author for Correspondence: Chen-Chou Lin.

*Associate Professor, Department of Mechanical and Marine Engineering, National Taiwan Ocean University, Keelung, Taiwan R.O.C. assign at most nine precision-points on the desired path for the synthesis of planar or spherical four-bar linkages. In practical design situation, one may desire to specify the ground pivot locations, or to coordinate between the crank angles and the position of the precision-points. In these cases, a maximum of five precision-points can be specified.

There are various approaches used in solving the planar motion and path generation problems, including graphical methods [1], analytical closed-form solutions [1], and numerical methods [2-11]. Graphical methods are used in simple cases or where high precision is not expected. Analytical closed-form solutions mainly refer to vector-loop method or complex-number method. It has been proved successful for them in solving path generation problems with five or less precision points. In the aspect of numerical method, Roth and Freudenstein [5] first proposed the Bootstrap approach in solving nine-point path generation problem for planar linkages. However, the approach does not guarantee the convergence of the problem. Nevertheless, the method has some similarities with continuation method. Recently, continuation method was applied in the synthesis of planar linkages, and proved to be an effective numerical technique $[4,8,10-12]$. The method has the advantages that it is a globally convergent numerical scheme which tend to be convergent, and users do not have to provide initial guess. Besides, the method can provide the complete set of solutions for the problems. The limitation of the method is that the total degree of the system can not be too large, otherwise the computation time would not be acceptable. In the past years, there were some investigations about the ways of reducing the system order for continuation method. For examples, introducing the multi-homogeneous variables, or parameter homotopy technique $[10,11]$, etc. We may obtain all of the mechanisms that meet the design specifications by continuation method. This means that mechanism designers can have more 
choices. On the other hand, traditional method such as Newton's method can provide only one set of solution. Often, even different initial guesses may converge to the same solution.

There were only limited research about the synthesis of spherical motion and path generation problems. Suh and Radcliffe [7] first applied displacement matrix in the synthesis of spherical path generators. However, the total degree of the design equations in their formulation become very large for five-point synthesis. Also they only solved the problem with moving pivots or fixed pivots locations specified. Recently, Chiang, Lin and Tong $[3,9]$ proposed the pole method which is considered as a semi graphical-analytical method. The formulation can handle various design specifications for path generation problems. We shall apply the concepts of graphical inversion and pole method to cast the design equations into the form of system of polynomial equations. The same examples in [9] will be illustrated and solved by continuation method here. The complete set of solutions will be provided for the synthesis of spherical linkages with five-position motion and path generation problems.

\section{THE DESIGN EQUATIONS}

If a rigid body undergoes a motion with the axis of rotation always passing through the center of a sphere $S$, then the rigid body motion is called a spherical motion. A spherical linkage is a linkage on which all of the points remain on concentric spherical surfaces during its movement. To solve the dimensional synthesis problems of planar mechanisms, researchers often model the linkages by vectors or complex numbers in their mathematical representations. However, for spatial or spherical linkages, we have to use matrices, or dual numbers/vectors to represent the linkages and their motion.

Consider a Cartesian coordinates system whose origin is located at the center $O$ of a unit sphere $S$. The equation of the spherical surface is then

$$
S: x^{2}+y^{2}+z^{2}=1 .
$$

Let the position vector of a point $P_{1}\left(x_{1}, y_{1}, z_{1}\right)$ be $\mathbf{r}_{1}$, and $\mathbf{r}_{1}$ rotate an angle $\phi$ about an axis of unit direction vector $\mathbf{u}$ to its second position $\mathbf{r}_{2}$. Then the spherical motion of the vector can be formulated by the Rodrigue equation as following:

$$
\mathbf{r}_{2}=(1-\cos \phi)\left(\mathbf{r}_{1} \cdot \mathbf{u}\right) \mathbf{u}+\cos \phi \mathbf{r}_{1}+\sin \phi\left(\mathbf{u} \times \mathbf{r}_{1}\right),
$$

or expressed in its matrix form as

$$
\mathbf{r}_{2}=R(\mathbf{u}, \phi) \mathbf{r}_{1},
$$

Where

$$
R(\mathbf{u}, \phi)=\left[\begin{array}{ccc}
u_{x}^{2} v \phi+c \phi & u_{x} u_{y} v \phi-u_{z} s \phi & u_{x} u_{z} v \phi+u_{y} s \phi \\
u_{x} u_{y} v \phi+u_{z} s \phi & u_{y}^{2} v \phi+c \phi & u_{y} u_{z} v \phi-u_{x} s \phi \\
u_{x} u_{z} v \phi-u_{y} s \phi & u_{y} u_{z} v \phi+u_{x} s \phi & u_{z}^{2} v \phi+c \phi
\end{array}\right],
$$

and $c \phi=\cos \phi, s \phi=\sin \phi, v \phi=1-\cos \phi$

To formulate the design equations of the motion generation (body guidance) and path generation problems of spherical four-bar linkages, here we shall use the concept of inversion in graphical synthesis approach of planar mechanisms, and the concept from the pole method developed by Chiang, Hsi, and Lin $[3,9]$. The formulation has the following advantages: (1) The problems can be formulated in the form of system equations of polynomials, which is required by continuation method, (2) The total degree of the polynomial system is far less than that of other formulation approach, such as that in [7], (3) In our approach, we may specify either ground pivot locations or crank angles for path generation problems. The second advantage is considered important since, in general, continuation method demands the system order be small. In what follows, we shall first review some of the above concepts, and modify the synthesis procedure in [9] in order to reduce the system's total degree to fit our need.

\section{Motion Generation (rigid-body guidance)}

We begin with the motion generation problem with four finitely separated positions specified. The problem is stated as following: If given four finitely separated positions, it is to be determined the locations of two fixed pivots $A_{0}, B_{0}$, and the first position of two moving pivots $A_{1}, B_{1}$ of linkages that can guide a body from position $1,2,3$, to position 4 . In general, the four positions can be specified in the form of displacement matrices, for example, $D_{1 i}$ denotes the transformation matrix between position 1 and $i$. Nevertheless, they can also be specified in the form of locations of two selected points on the coupler. And, the later specifications can always be transformed into the first one. Now let the homologous points of the circle-point $A_{1}$ be denoted by $A_{i}(i=2,3,4)$, we then have the following relation:

$$
\left[\begin{array}{l}
x_{A i} \\
y_{A i} \\
z_{A i}
\end{array}\right]=D_{1 i}\left[\begin{array}{l}
x_{A 1} \\
y_{A 1} \\
z_{A 1}
\end{array}\right] . \quad i=2,3,4 .
$$


Because all circle-points $A_{i}$ must lie on the circle centered at the center-point $A_{0}$, meaning that $A_{0}\left(x_{A 0}\right.$, $\left.y_{A 0}, z_{A 0}\right)$ must lie on the plane of perpendicular bisector between $A_{1}$ and $A_{i}$. Therefore, we may obtain the following equations:

$$
\begin{gathered}
\left(x_{A i}-x_{A 1}\right) x_{A 0}+\left(y_{A i}-y_{A 1}\right) y_{A 0}+\left(z_{A i}-z_{A 1}\right) z_{A 0}=0 \\
i=2,3,4 .
\end{gathered}
$$

In its matrix form, we have

$$
\begin{gathered}
{\left[\begin{array}{ccc}
x_{A 2}-x_{A 1} & y_{A 2}-y_{A 1} & z_{A 2}-z_{A 1} \\
x_{A 3}-x_{A 1} & y_{A 3}-y_{A 1} & z_{A 3}-z_{A 1} \\
x_{A 4}-x_{A 1} & y_{A 4}-y_{A 1} & z_{A 4}-z_{A 1}
\end{array}\right]\left[\begin{array}{l}
x_{A 0} \\
y_{A 0} \\
z_{A 0}
\end{array}\right]=0 .} \\
i=2,3,4 .
\end{gathered}
$$

Substitute Eq. (4) into Eq. (6), we get

$$
\left[\begin{array}{lll}
U_{1} & V_{1} & W_{1} \\
U_{2} & V_{2} & W_{2} \\
U_{3} & V_{3} & W_{3}
\end{array}\right]\left[\begin{array}{l}
x_{A 0} \\
y_{A 0} \\
z_{A 0}
\end{array}\right]=0
$$

where $U_{i}=\left(d_{11 i}-1\right) x_{A 1}+d_{12 i} y_{A 1}+d_{13 i} z_{A 1}$,

$$
\begin{aligned}
& V_{i}=d_{21 i} x_{A 1}+\left(d_{22 i}-1\right) y_{A 1}+d_{23 i} z_{A 1}, i=1,2 \\
& W_{i}=d_{31 i} x_{A 1}+d_{32 i} y_{A 1}+\left(d_{33 i}-1\right) z_{A 1}
\end{aligned}
$$

and $d_{p q i}$ denotes the matrix element in $D_{1 i}$.

For the existence of non-trivial solutions of $A_{0}$ in Eq. (7), the determinant of the square matrix of left-hand side must vanish. By expanding the determinant, we derive the equation of a spherical cubic cone, whose intersection with the unit sphere $S$ represents the circle-point curve, which is denoted by $k_{1}$. The expanded determinant is expressed in terms of polynomials of $x_{A 1}, y_{A 1}, z_{A 1}$ as following:

$$
\begin{aligned}
k_{1}: & c_{1} x_{A 1}^{3}+c_{2} y_{A 1}^{3}+c_{3} z_{A 1}^{3}+c_{4} x_{A 1}^{2} y_{A 1} \\
& +c_{5} x_{A 1}^{2} z_{A 1}+c_{6} y_{A 1}^{2} x_{A 1}+c_{7} y_{A 1}^{2} z_{A 1} \\
& +c_{8} z_{A 1}^{2} x_{A 1}+c_{9} z_{A 1}^{2} y_{A 1}+c_{10} x_{A 1} y_{A 1} z_{A 1}=0,
\end{aligned}
$$

where the coefficients $c_{i}$ 's are not listed here for brevity. In practice, we use symbolic processing software package Mathematica to derive these coefficients.

For the four-position motion generation problem, we may arbitrarily assign a coordinate, for example, $x_{A 1}$, substitute it into Eq. (9), then solve together with Eq. (1), to get the $y_{A 1}$, and $z_{A 1}$. Then we substitute these coordinates into two of the equations in Eq. (7), together with Eq. (1), we may now get the coordinates of the corresponding center-point $A_{0}$. Similarly, we can solve for the coordinates of the other Burmester-point pair $B_{1}$ and $B_{0}$. Hence we may conclude that there are $\infty$ number of free choices for the synthesis of four-position motion generation of spherical four-bar linkages.

For five finitely separated position motion generation problem, we are given by the displacement matrices $D_{1 i}, i=2$ to 5 . Following the same procedure as in four-position problem, we may derive two equations of the spherical cubic cones $k_{1(1234)}$ and $k_{1(1235)}$. Together with Eq. (1), we may solve for the locations of circle points $A_{1}$ and $B_{1}$, and their corresponding center points $A_{0}$ and $B_{0}$. There is no free choice in the synthesis five-position motion generation problems; meaning that there are only finite number of linkages to be synthesized in this kind of problems. It has been pointed out by Roth in [6], that there are at most six real solutions (excluding their origin symmetric solutions) for spherical five-position motion generation problems.

\section{Path Generation with Prescribed Crank Angles}

The path generation problem with prescribed crank angles is stated as following: Given the locations of four discrete points, $E_{i}(i=1$ to 4$)$, and three crank angles, $\phi_{2}, \phi_{3}$ and $\phi_{4}$, it is required to synthesize linkages whose coupler point can pass the four precision points, and coordinate with respect to the prescribed crank angles. Here we shall apply the concept of inversion process in graphical synthesis. Fig. (1a) shows the prescribed crank angles, the precision points, as well as the linkage to be determined. If we fix the crank $A_{0} A_{1}$, the corresponding positions of the four precision points with respect the crank angles become $E_{i}^{\prime}(i=1$ to 4$)$. The positions of $E^{\prime}$ is can be obtained by rotating $A_{0} E_{i}$ about $A_{0}$ axis through $-\phi_{2},-\phi_{3}$ and $-\phi_{4}$, as shown in Fig. (1b), and their coordinates can be expressed as .

$$
\left[\begin{array}{c}
x_{\mathrm{Ei}}^{\prime} \\
y_{\mathrm{Ei}}^{\prime} \\
z_{\mathrm{Ei}}^{\prime}
\end{array}\right]=R\left(\overrightarrow{O A_{0}},-\phi_{i}\right)\left[\begin{array}{c}
x_{\mathrm{Ei}} \\
y_{\mathrm{Ei}} \\
z_{\mathrm{Ei}}
\end{array}\right] . \quad i=2,3,4
$$

Equation (10) shows that the coordinates of $E_{i}$ can be expressed in terms of polynomials of $x_{A 0}, \mathrm{y}_{A 0}$, $z_{A 0}$. Since points $E_{1}, E_{2}^{\prime}, E_{3}^{\prime}$, and $E_{4}^{\prime}$ must locate on the circle centered at $A_{1}$, it follows that

$$
\begin{gathered}
\left(x_{\mathrm{Ei}}^{\prime}-x_{E 1}\right) x_{A 1}+\left(y_{\mathrm{Ei}}^{\prime}-y_{E 1}\right) y_{A 1}+\left(z_{\mathrm{Ei}}^{\prime}-z_{E 1}\right) z_{A 1}=0 \\
i=2,3,4
\end{gathered}
$$

For the existence of nonzero solution of $x_{A 1}, y_{A 1}$, 

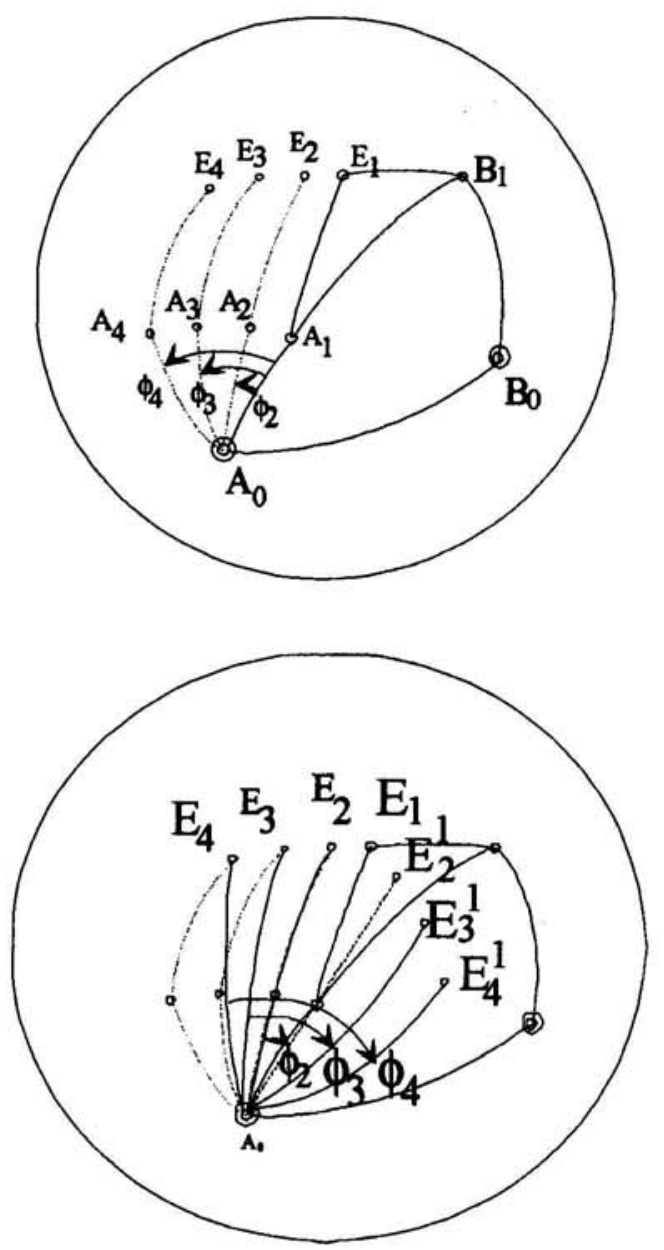

Fig. 1. (A) Four-Point Path Generation Problem. (B) An Inversion of Fig. 1(A).

and $z_{A 1}$ in the homogeneous equations set Eq. (11), the determinant of the coefficient matrix must vanish. Hence .

$$
\operatorname{det}\left(\left[\begin{array}{ccc}
x_{E 2}^{\prime}-x_{E 1} & y_{E 2}^{\prime}-y_{E 1} & z_{E 2}^{\prime}-z_{E 1} \\
x_{E 3}^{\prime}-x_{E 1} & y_{E 3}^{\prime}-y_{E 1} & z_{E 3}^{\prime}-z_{E 1} \\
x_{E 4}^{\prime}-x_{E 1} & y_{E 4}^{\prime}-y_{E 1} & z_{E 4}^{\prime}-z_{E 1}
\end{array}\right]\right)=0 .
$$

By expanding the determinant of Eq. (12), we obtain a sixth-degree polynomial equation in variables of $x_{A 0}, y_{A 0}, z_{A 0}$. We may further reduce it into a third-degree polynomial equation, if a proper coordinate system [13] is chosen. The intersection of the surface with a unit sphere $S$ is a cubic-order curve, which represents the collection of the center-points $A_{0}$ that satisfies the design specifications. It is expressed as following:

$$
k_{A 0}: k_{31} x_{A 0}^{3}+k_{32} y_{A 0}^{3}+k_{33} z_{A 0}^{3}+k_{34} x_{A 0}^{2} y_{A 0}
$$

$$
\begin{aligned}
& +k_{35} x_{A 0}^{2} z_{A 0}+k_{36} y_{A 0}^{2} z_{A 0}+k_{37} y_{A 0}^{2} x+k_{38} z_{A 0}^{2} x_{A 0} \\
& +k_{39} z_{A 0}^{2} y_{A 0}+k_{30} x_{A 0} z_{A 0}+k_{21} x_{A 0}^{2}+k_{22} y_{A 0}^{2} \\
& +k_{23} z+k_{24} x_{A 0} y_{A 0}+k_{25} y_{A 0} z_{A 0}+k_{26} x_{A 0} z_{A 0} \\
& +k_{11} x_{A 0}+k_{12} y_{A 0}+k_{13} z_{A 0}+k_{0}=0 .
\end{aligned}
$$

where the coefficients $k_{p q}$ are not listed here for brevity, nevertheless, they can be obtained by using symbolic processing package. It is shown that $k_{p q}$ 's are functions of positions of precision points and the prescribed crank angles.

For the four-position path generation problem, we may arbitrarily assign a coordinate, for example, $x_{A 0}$, substituting it into Eq. (13), then solve together with Eq. (1), to get the $y_{A 0}$, and $z_{A 0}$. Then we substitute these coordinates into Eq. (10) to get coordinates of $E_{s}^{\prime}$. Then solve two of the equations in Eq. (11), together with Eq. (1), we may now get the location of the corresponding circle-point $A_{1}$. To solve the positions of the other pair of pivots $B_{1}$ and $B_{0}$, we first find the coordinates of homologous points $A_{2}, A_{3}$, and $A_{4}$, then derive the coupler's displacement matrix $D_{1 i}$ from positions of $A_{i} E_{i}(i=1,2,3,4)$. At this stage, we may follow the procedure in motion generation synthesis to solve the positions of $B_{1}$ and $B_{0}$. As we may see, there are also $\infty$ free choices in the design process.

For five-point path generation problem, as in four-point problem, we may derive the equations of the center-point $A_{0}, k_{A 0 \text { (1234) }}$ and $k_{A 0(1235)}$, together with Eq. (1), solve for the locations of the center points $A_{0}$. Note that the solution number of $A_{0}$ is finite here. Then the corresponding circle-point $A_{1}$ and their homologous points $A_{2}, A_{3}, A_{4}$, and $A_{5}$ can be found. At this stage, we may follow the steps in fiveposition motion generation problem. By solving the equations $k_{B 1(1234)}$ and $k_{B 1(1235)}$, together with Eq. (1), we get the locations of the other circle points $B_{1}$. Finally, the corresponding center point $B_{0}$ can be derived accordingly.

\section{Path Generation with Ground Pivot Specifications}

In this case, the prescribed specifications are the positions of two fixed pivots, $A_{0}$ and $B_{0}$, along with the precision points $E_{i}(i=1,2,3,4,5)$. We shall apply the concept of pole method proposed by Chiang [9] in order to find the positions of moving pivots $A_{1}$ and $B_{1}$. However, here we make some modifications in order to shape our formulations into polynomial form. Figure 2 shows the spherical linkage in its first and $i$ th positions, and $A_{0} A_{1}$ and $B_{0} B_{1}$ subtending equal angles $\gamma_{i} / 2$ at the pole $P_{i}$. If we rotate the position vector $\overrightarrow{O A_{0}}$ about the ith pole axis $\overrightarrow{O P}_{i}$, through the 


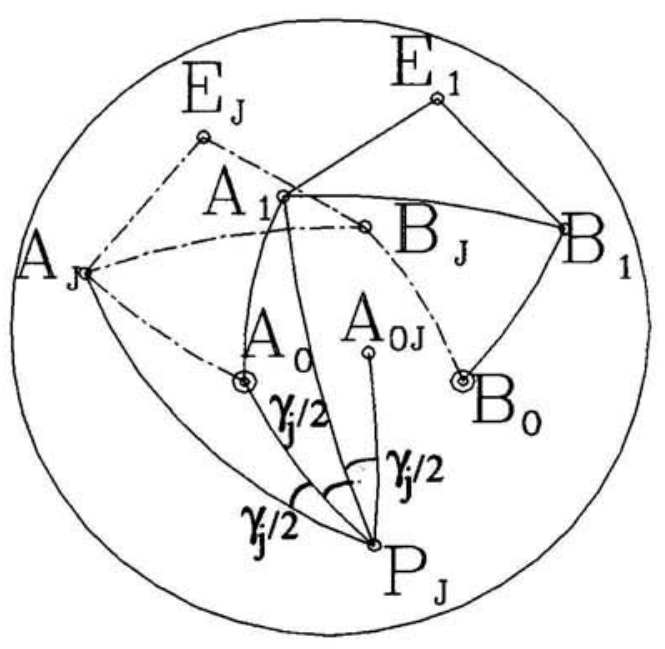

Fig. 2. The Angles Subtended by $\mathrm{A}_{\mathrm{j}} \mathrm{A}_{0}$ and $\mathrm{A}_{\mathrm{j}} \mathrm{A}_{1}$ at Pole.

negative ith angle of rotation of the coupler $-\gamma_{i}$, we obtain a second position $\overrightarrow{O A_{0 i}}(i=2,3,4,5)$. It is clear that $A_{1}$ lies on the plane of perpendicular bisector between $A_{0}$ and $A_{0 i}$. Therefore, we have the following relations:

$$
\begin{gathered}
\left(x_{A 0 i}-x_{A 0}\right) x_{A 1}+\left(y_{A 0 i}-y_{A 0}\right) y_{A 1}+\left(z_{A 0 i}-z_{A 0}\right) z_{A 1} \\
=0 . \quad i=2,3,4,5 .
\end{gathered}
$$

Similarly, for the other fixed pivot $B_{0}$, we have

$$
\begin{gathered}
\left(x_{B 0 i}-x_{B 0}\right) x_{B 1}+\left(y_{B 0 i}-y_{B 0}\right) y_{B 1}+\left(z_{B 0 i}-z_{B 0}\right) z_{B 1} \\
=0 . \quad i=2,3,4,5 .
\end{gathered}
$$

For the existence of nonzero solution of $x_{A 1}, \mathrm{y}_{A 1}$, $\mathrm{z}_{A 1}, x_{B 1}, y_{B 1}$, and $z_{B 1}$ of the above homogeneous equations set, the following determinant has to vanish. That is,

$$
\begin{aligned}
& \operatorname{det}\left(\left[\begin{array}{lll}
x_{A 02}-x_{A 0} & y_{A 02}-y_{A 0} & z_{A 02}-z_{A 0} \\
x_{A 03}-x_{A 0} & y_{A 03}-y_{A 0} & z_{A 03}-z_{A 0} \\
x_{A 04}-x_{A 0} & y_{A 04}-y_{A 0} & z_{A 04}-z_{A 0}
\end{array}\right]\right)=0, \\
& \operatorname{det}\left(\left[\begin{array}{lll}
x_{A 03}-x_{A 0} & y_{A 03}-y_{A 0} & z_{A 03}-z_{A 0} \\
x_{A 04}-x_{A 0} & y_{A 04}-y_{A 0} & z_{A 04}-z_{A 0} \\
x_{A 05}-x_{A 0} & y_{A 05}-y_{A 0} & z_{A 05}-z_{A 0}
\end{array}\right]\right)=0, \\
& \operatorname{det}\left(\left[\begin{array}{lll}
x_{B 02}-x_{B 0} & y_{B 02}-y_{B 0} & z_{B 02}-z_{B 0} \\
x_{B 03}-x_{B 0} & y_{B 03}-y_{B 0} & z_{B 03}-z_{B 0} \\
x_{B 04}-x_{B 0} & y_{B 04}-y_{B 0} & z_{B 04}-z_{B 0}
\end{array}\right]\right)=0,
\end{aligned}
$$

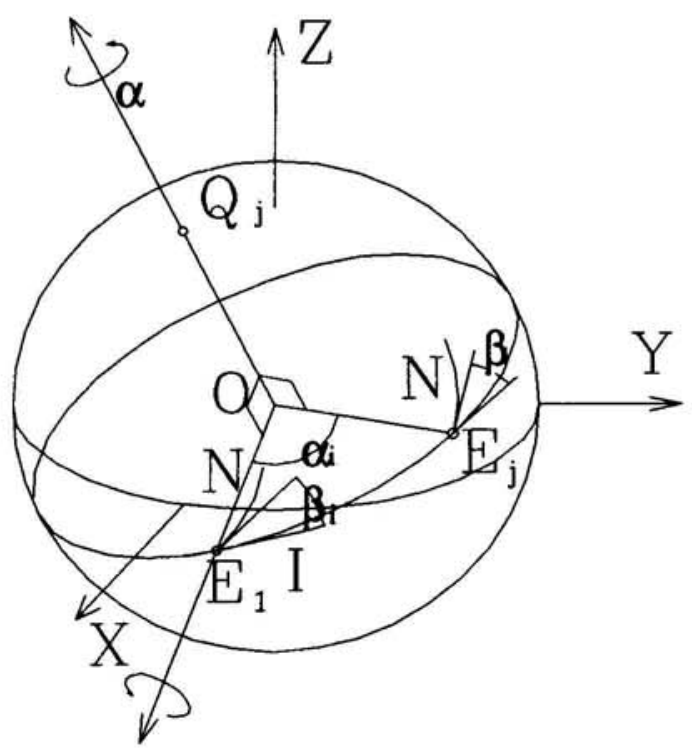

Fig. 3. Two Success Rotations By the Coupler.

$$
\operatorname{det}\left(\left[\begin{array}{lll}
x_{B 03}-x_{B 0} & y_{B 03}-y_{B 0} & z_{B 03}-z_{B 0} \\
x_{B 04}-x_{B 0} & y_{B 04}-y_{B 0} & z_{B 04}-z_{B 0} \\
x_{B 05}-x_{B 0} & y_{B 05}-y_{B 0} & z_{B 05}-z_{B 0}
\end{array}\right]\right)=0
$$

In Chiang's approach, he used the following Rodrigue equations to express the coordinates of $A_{0 i}$ (or $B_{0 i}$ ) in Eq. (16) to Eq. (19).

$$
\begin{aligned}
\mathbf{r}_{A 0 i} & =\left(1-\cos \gamma_{i}\right)\left(\mathbf{r}_{P i} \cdot \mathbf{r}_{A 0}\right) \mathbf{r}_{P i}+\cos \gamma_{i} \mathbf{r}_{A 0} \\
& -\sin \gamma_{i}\left(\mathbf{r}_{P i} \times \mathbf{r}_{A 0}\right),
\end{aligned}
$$

with $\mathbf{r}_{P i}$ expressed in terms of $\cos \gamma_{i}, \sin \gamma_{i}$, and coordinates of $E_{i}$. However, it becomes tedious when we try to convert it into polynomial form, because that $\cos \gamma_{i}$ term is contained in the square-root's bracket. We shall use the displacement matrix $D_{1 i}$ in [7] to express the coordinates of $A_{0 i}$ and $B_{0 i}$ Let the displacement matrix be product of two rotation matrices, as shown in Fig. (3). ,

$$
D_{1 i}=R\left(\mathbf{Q}_{i}, \alpha_{i}\right) R\left(\mathbf{E}_{i}, \beta_{i}\right)
$$

where $\mathbf{Q}_{i}=\mathbf{E}_{1} \times \mathbf{E}_{i}$, and $\alpha_{i}$ is the angle between the position vectors of the first and ith precision points. Therefore,

$$
\begin{aligned}
& {\left[\begin{array}{l}
x_{A 0 i} \\
y_{A 0 i} \\
z_{A 0 i}
\end{array}\right]=D_{1 i}^{-1}\left[\begin{array}{l}
x_{A 0} \\
y_{A 0} \\
z_{A 0}
\end{array}\right]=R\left(\mathbf{E}_{i},-\beta_{i}\right) R\left(\mathbf{Q}_{i},-\alpha_{i}\right)\left[\begin{array}{l}
x_{A 0} \\
y_{A 0} \\
z_{A 0}
\end{array}\right] .} \\
& i=2,3,4,5
\end{aligned}
$$


It is shown from Eq. (21) that $x_{A 0 i}, y_{A 0 i}$, and $z_{A 0 i}$, can be expressed in linear combinations of $\cos \beta_{i}$, and $\sin \beta_{i}$. Similarly, $x_{B 0 i}, y_{B 0 i}$, and $z_{B 0 i}$ can be derived in the same manner.

$$
\left[\begin{array}{l}
x_{B 0 i} \\
y_{B 0 i} \\
z_{B 0 i}
\end{array}\right]=R\left(\mathbf{E}_{i},-\beta_{i}\right) R\left(\mathbf{Q}_{i},-\alpha_{i}\right)\left[\begin{array}{l}
x_{B 0} \\
y_{B 0} \\
z_{B 0}
\end{array}\right] .
$$$$
i=2,3,4,5 \text {. }
$$

We may now substitute right-hand side of Eqs. (21) and (22) into Eqs. (16), (17), and (18), (19), respectively, then expand the determinants. Hence Eqs. (16) and (17) become

$$
\begin{aligned}
L^{A}{ }_{234}: & m_{31} s_{2} s_{3} s_{4}+m_{32} s_{2} s_{3} c_{4}+m_{33} s_{2} c_{2} s_{4}+m_{34} s_{2} c_{3} c_{4} \\
& +m_{35} c_{2} s_{3} s_{4}+m_{36} c_{2} s_{3} c_{4}+m_{37} c_{2} c_{3} s_{4}+m_{38} c_{2} c_{3} c_{4} \\
& +m_{21} s_{2} s_{2}+m_{22} s_{2} c_{3}+m_{23} s_{2} s_{4}+m_{24} s_{2} c_{4}+m_{25} c_{2} s_{3} \\
& +m_{26} c_{2} c_{3}+m_{27} c_{2} s_{4}+m_{28} c_{2} c_{4}+m_{29} s_{3} s_{4} \\
& +m_{2,10} s_{3} c_{4}+m_{2,11} c_{3} s_{4}+m_{2,12} c_{3} c_{4}+m_{11} s_{2} \\
& +m_{12} c_{2}+m_{13} s_{3}+n_{14} c_{3}+m_{15} s_{4}+m_{16} c_{4}+m_{0} \\
& =0,
\end{aligned}
$$

$$
\begin{aligned}
L^{A}{ }_{345}: & n_{31} s_{3} s_{4} s_{5}+n_{32} s_{3} s_{4} c_{5}+n_{33} s_{3} c_{4} s_{5}+n_{34} s_{3} c_{4} c_{5} \\
& +n_{35} c_{3} s_{4} s_{5}+n_{36} c_{3} s_{4} c_{5}+n_{37} c_{3} c_{4} s_{5}+n_{38} c_{3} c_{4} c_{5} \\
& +n_{21} s_{3} s_{3}+n_{22} s_{3} c_{4}+n_{23} s_{3} s_{5}+n_{24} s_{3} c_{5}+n_{25} c_{3} s_{4} \\
& +n_{26} c_{3} c_{4}+n_{27} c_{3} s_{5}+n_{28} c_{3} c_{5}+n_{29} s_{3} s_{5}+n_{2,10} s_{4} c_{5} \\
& +n_{2,11} c_{4} s_{5}+n_{2,12} c_{4} c_{5}+n_{11} s_{3}+n_{12} c_{3}+m_{13} s_{4} \\
& +n_{14} c_{4}+n_{15} s_{4}+n_{16} c_{5}+n_{0}=0,
\end{aligned}
$$

Similarly, equations (18) and (19) also have the same form of Eqs. (23) and (24),

$$
\begin{aligned}
& L^{B}{ }_{234}: m_{31}^{\prime} s_{2} s_{3} s_{4}+m_{32}^{\prime} s_{2} s_{3} c_{4}+\ldots+m_{0}^{\prime}=0, \\
& L^{B}{ }_{345}: n_{31}^{\prime} s_{3} s_{4} s_{5}+n_{32}^{\prime} s_{3} s_{4} c_{5}+\ldots+n_{0}^{\prime}=0,
\end{aligned}
$$

where $s_{i}=\sin \beta_{i}$ and $c_{i}=\cos \beta_{i}, i=2,3,4,5$. The coefficients $m_{p q}$ 's and $n_{p q}$ 's can be derived by symbolic processing packages, and are not listed here for brevity.

To solve the variables $\beta_{i}$, we need four more compatibility equations:

$$
C_{i}: \sin ^{2} \beta_{i}+\cos ^{2} \beta_{i}-1=0 . \quad i=2,3,4,5 .
$$

Once $\beta_{i}$ are solved, we may substitute them into Eqs. (21) and (22) to get the coordinates of $A_{0 i}$ and $B_{0 i}$. Finally, we substitute these coordinates into two of the Eqs. set (14) and (15), together with Eq. (1), we may solve the positions of moving pivots $A_{1}$ and $B_{1}$.

NUMERICAL CONTINUATION
The principle of continuation method (or homotopy method) is that we begin with a start system whose solutions are known, and each polynomial equation in the start system have the same degree as that of the corresponding polynomial equation in the target system. Then we track from the initial point along a homotopy path, if the path does not diverge, we shall achieve the target system with the problem solved. During the process of path tracking, we are actually solving many subproblems by Newton method (or other locally-convergent method); Specifically, the solution of previous homotopy step is used as the initial guessing values of the current step. In the traditional homotopy method, the homotopy function $\mathbf{H}$ is defined as:

$$
\mathbf{H}(\mathbf{z}, t): \quad t \mathbf{F}(\mathbf{z})+(1-\mathrm{t}) \mathbf{G}(\mathbf{z}) .
$$

where $t$ is the homotopy parameter. When $t$ equals 0 , the homotopy function $\mathbf{H}$ is the same as the start system $\mathbf{G}(z)$; and when $t$ equals $1, \mathbf{H}$ becomes the system polynomial equations $\mathbf{F}$ we want to solve. Note that $\mathbf{H}, \mathbf{G}, \mathbf{F}$, and $\mathbf{z}$ are arrays of the same dimension. The start system $\mathbf{G}$ is generally defined as the following:

$$
G_{i}: C_{i 1} z_{i}^{d_{i}}-C_{i 2}=0 . \quad i=1,2, \ldots ., n .
$$

where $C_{i 1}$ and $C_{i 2}$ are random numbers, $d_{i}$ is the degree of the equation $F_{i}$.

The tracked path is called homotopy path. The number of total homotopy paths is the product of each polynomial equation's degree of the system $\mathbf{F}$, or is called Bezout number. The way of following the homotopy path incrementally from $t=0$ to 1 is called path-tracking. There are three path-tracking algorithms mentioned in the literature. They are: (1) Ordinary Differential Equation Based, (2) Normal Flow, (3) Augmented Jacobian Matrix, denoted by $\mathrm{DF}, \mathrm{NF}$, and QF, respectively. Here we adopt the NF algorithm in computation.

Suppose we want to track a homotopy path, and its initial value $\left(z_{0}, t_{0}\right)$ is known. We first take an increment, and along the tangential direction of the homotopy path at $t=t_{0}$, we may obtained a predicted value of $\mathbf{z}$ at $t=t_{0}+\Delta t$ by

$$
\mathbf{z}=\left(t=t_{0}+\Delta t\right)=\mathbf{z}\left(t_{0}\right)+(d \mathbf{z} / d t) \Delta t
$$

The unit tangential vector $d \mathbf{z} / d t$ can be obtained by first taking derivative of $\mathbf{H}$ with respect to $t$, that is:

$$
\frac{d \mathbf{H}(z(t), t)}{d \mathbf{z}} \frac{d \mathbf{z}}{d t}=-\mathbf{H}_{t}(z(t), t) .
$$


In Eq. (31), $\frac{d \mathbf{H}(z(t), t)}{d \mathbf{z}}$ is an $n \times n$ matrix, $\mathbf{H}_{t}(z(t)$, $t)$ is an $n \times 1$ array. We may use QR decomposition method to solve the above linear equation system, and obtain $d \mathbf{z} / d t$. Note that $d \mathbf{z} / d t$ in Eq. (30) is a unit vector, so that we have to normalize the vector obtained from Eq. (31). After the predicted value is obtained, we use Newton method to compute the correct value at $t=t_{0}+\Delta t$. Let $\mathbf{T}(\mathbf{z})=\mathbf{H}\left(\mathbf{z}, t_{0}+\Delta t\right)$

$$
\begin{aligned}
& \mathbf{z}_{0}=\hat{\mathbf{z}}, \\
& \mathbf{z}_{k+1}=\mathbf{z}_{k}-\left(\frac{d \mathbf{T}\left(\mathbf{z}_{k}\right)}{d \mathbf{z}}\right)^{-1} \mathbf{T}\left(\mathbf{z}_{k}\right),
\end{aligned}
$$

where $\hat{\mathbf{z}}$ is the predicted value obtained from Eq. (30).

In practical implementation of the scheme, we have to evaluate the angle between the tangential vectors at $(\mathbf{z}(t), t)$ and $(\mathbf{z}(t+\Delta t), \mathrm{t}+\Delta t)$. This is to avoid the situation of abrupt function value change between the current and next step. If abrupt change do occur, we then restart the predicting process by taking a smaller step $(\Delta t / 2)$.

\section{NUMERICAL EXAMPLES}

In section 2 we've cast the design equations of three types of problems into system of polynomial equations. Here we shall apply the continuation method to solve the problems. A motion generation problem is imbedded in a problem of path generation with prescribed crank angles, therefore, it will not be illustrated individually. Two examples will be given. path generation with prescribed crank angles. To synthesize a five-point path generation problem with prescribed crank angles, we first determine the location of $A_{0}$ by solving $k_{A 0(1234)}$ and $k_{A 0(1235)}$ of Eq. (13) and Eq. (1). After the locations of $A_{1}, A_{2}, \ldots A_{5}$ are obtained, we then solve the coordinates of the other circle-point $B_{1}$ by solving $k_{B 1(1234)}$ and $k_{B 1(1235)}$ of Eq.
Table 1. The coordinates of precision points and corresponding crank angles

\begin{tabular}{clccc}
\hline$E_{i}$ & \multicolumn{1}{c}{$x_{i}$} & $y_{i}$ & $z_{i}$ & $\phi_{i}$ (deg.) \\
\hline 1 & 0 & 0.35157691 & 0.936159 & 0 \\
2 & -0.120247 & 0.33037401 & 0.936159 & 20 \\
3 & -0.220407 & 0.25632520 & 0.941126 & 40 \\
4 & -0.286565 & 0.12486548 & 0.949889 & 60 \\
5 & -0.306167 & -0.01378554 & 0.951878 & 75 \\
\hline
\end{tabular}

(9) and Eq. (1) simultaneously. Finally, the location of $B_{0}$ are determined. Note that the Bezout numbers of both subproblems are 18 .

\section{Example 1}

The coordinates of five precision points $E_{i}$ and four crank angles $\phi_{i}$ are shown in Table 1. The example is taken from Chiang's example [9], but with higher precision. We apply the traditional homotopy to solve $k_{A 0(1234)}$ and $k_{A 0(1235)}$ of Eq. (13) and Eq. (1). We found ten sets of real solutions of $A_{0}$, as listed in Table 2. Then the corresponding circle-point $A_{1}$ is derived by solving two of the Eq. (11) and Eq. (1), which is also listed in Table 2. By finding the homologous points of $A_{1}$, together with the coordinates of precision points $E_{i}(i=1, \ldots, 5)$, we may solve the coordinates of the other circle-point $B_{0}$ (an imbedded motion generation problem). Again we apply the traditional homotopy to solve $k_{B 1(1234)}$ and $k_{B 1(1235)}$ of Eq. (9) and Eq. (1). Ten sets of real solutions of $B_{1}$ are found, but only five sets are shown in Table 3 due to the origin symmetry property. Finally, their corresponding center-point $B_{0}$ can be obtained by solving two of the Eq. (5) and Eq. (1), as shown in Table 3. Note that not all of the corresponding center-point of $B_{1}$ can be obtained due to the fact that some solutions of $B_{1}$ are at the poles position.

Table 2. The real solutions of $A_{0}$ and their corresponding $A_{1}$

\begin{tabular}{clrrrrc}
\hline & \multicolumn{1}{c}{$x_{A 0}$} & \multicolumn{1}{c}{$y_{A 0}$} & \multicolumn{1}{c}{$z_{A 0}$} & \multicolumn{1}{c}{$x_{A 1}$} & \multicolumn{1}{c}{$y_{A 1}$} & $z_{A 1}$ \\
\hline 1 & 0.1298623 & -0.7421495 & 0.6575332 & 0.1396759 & -0.2188076 & 0.9657193 \\
2 & 0.2314213 & -0.5763645 & -0.7837399 & 0.0899787 & 0.4466046 & 0.8901956 \\
3 & 0 & 0 & 1 & -0.0843698 & 0.3653350 & 0.9270448 \\
4 & 0.7645452 & 0.1203288 & 0.6332390 & 0.7669234 & 0.4366295 & 0.4703013 \\
5 & 0.0424124 & 0.0096460 & 0.9990536 & -0.0412003 & 0.3562823 & 0.9334696 \\
6 & 0.1129917 & -0.6408044 & -0.7593435 & 0.1323077 & 0.4230299 & 0.8964042 \\
7 & 0.0220257 & 0.0011671 & 0.9997567 & -0.0645178 & 0.3610775 & 0.9303013 \\
8 & 0.3616052 & -0.5400912 & -0.7599626 & 0.0281881 & 0.4161886 & 0.9088413 \\
9 & -0.2884520 & 0.2863784 & -0.9136645 & 0.1684800 & -0.2483078 & 0.9539170 \\
10 & -0.1186018 & 0.1194402 & 0.9857320 & -0.2160792 & 0.5737059 & 0.7900451 \\
\hline
\end{tabular}


Table 3. The real solutions of $B_{1}$ and $B_{0}$ corresponding to solution set \#1 in Table 2

\begin{tabular}{rrrrrrc}
\hline & $x_{B 1}$ & \multicolumn{1}{c}{$y_{B 1}$} & $z_{B 1}$ & $x_{B 0}$ & $y_{B 0}$ & $z_{B 0}$ \\
\hline 1 & 0.4423878 & 0.6333900 & 0.6349095 & 0.8976987 & 0.0161331 & 0.4403144 \\
2 & -0.3025020 & 0.7318484 & 0.6106476 & & $B_{1}$ is the pole $P_{13}$ & \\
3 & 0.1396759 & -0.2188077 & 0.9657193 & 0.1298623 & -0.7421495 & 0.6575332 \\
4 & -0.4319020 & 0.6243112 & 0.6509195 & & $B_{1}$ is the pole $P_{23}$ & \\
5 & -0.1415574 & 0.8027400 & 0.5792841 & & $B_{1}$ is the pole $P_{12}$ & \\
\hline
\end{tabular}

Table 4. The nondegenerate mechanisms fulfilling the design specifications in Ex. 1

\begin{tabular}{|c|c|c|c|c|c|}
\hline & $\mathrm{A}_{0}$ & $\mathrm{~A}_{1}$ & $\mathrm{~B}_{0}$ & $\mathrm{~B}_{1}$ & Comments \\
\hline 1 & $\begin{array}{l}(0.129862265, \\
-0.742149525, \\
0.657533174)\end{array}$ & $\begin{array}{l}(0.139675922, \\
-0.218807698, \\
0.965719332)\end{array}$ & $\begin{array}{l}0.897698706 \\
0.016133077 \\
0.440314385)\end{array}$ & $\begin{array}{c}(0.442387619 \\
0.633390289 \\
0.634909393)\end{array}$ & $\begin{array}{c}\text { Grashof } \\
\text { link2 is crank }\end{array}$ \\
\hline 2 & $\begin{array}{l}(0.231421280, \\
-0.576364472, \\
-0.783739872)\end{array}$ & $\begin{array}{l}(0.089978658, \\
0.446604573, \\
0.890195594)\end{array}$ & $\begin{array}{c}(-0.254837656 \\
0.095291287 \\
0.962277164)\end{array}$ & $\begin{array}{c}(-0.107934214 \\
0.189237532 \\
0.975981230)\end{array}$ & $\begin{array}{c}\text { Grashof } \\
\text { link2 is rocker } \\
\text { order defect }\end{array}$ \\
\hline 3 & $\begin{array}{l}(0.231421280, \\
-0.576364472, \\
-0.783739872)\end{array}$ & $\begin{array}{c}(0.089978658 \\
0.446604573 \\
0.890195594)\end{array}$ & $\begin{array}{c}(0.007059200 \\
0.073698997 \\
0.997255547)\end{array}$ & $\begin{array}{c}(0.003638768 \\
0.360721754 \\
0.932666380)\end{array}$ & Non-Grashof \\
\hline 4 & $\begin{array}{l}(0.231421280, \\
-0.576364472, \\
-0.783739872)\end{array}$ & $\begin{array}{l}(0.089978658 \\
0.446604573 \\
0.890195594)\end{array}$ & $\begin{array}{c}(0.460338718 \\
-0.522163799 \\
0.717936788)\end{array}$ & $\begin{array}{c}(-0.000057737 \\
0.434473531 \\
0.900684599)\end{array}$ & Non-Grashof \\
\hline 5 & $(0,0,1)$ & $\begin{array}{c}(-0.084369834 \\
0.365335035 \\
0.927044790)\end{array}$ & $\begin{array}{c}(0.530088022, \\
0.105575252 \\
-0.841344493)\end{array}$ & $\begin{array}{c}(-0.311846322 \\
-0.006881392 \\
0.950107635)\end{array}$ & Non-Grashof \\
\hline 6 & $\begin{array}{l}(0.764545218, \\
0.120328843, \\
0.633238959)\end{array}$ & $\begin{array}{l}(0.766923357 \\
0.436629458 \\
0.470301265)\end{array}$ & $\begin{array}{l}(0.898300301, \\
-0.146008413, \\
0.414412973)\end{array}$ & $\begin{array}{c}(0.930717582 \\
0.303664839 \\
0.203844174)\end{array}$ & Non-Grashof \\
\hline 7 & $\begin{array}{l}(0.042412350 \\
0.009646048, \\
0.999053625)\end{array}$ & $\begin{array}{c}(-0.041200305 \\
0.356282301 \\
0.933469580)\end{array}$ & $\begin{array}{c}(0.029974548 \\
0.022777476 \\
0.999291105)\end{array}$ & $\begin{array}{c}(-0.035183231 \\
0.413319935 \\
0.909905914)\end{array}$ & Non-Grashof \\
\hline 8 & $\begin{array}{l}(0.112991721, \\
-0.640804412, \\
-0.759343516)\end{array}$ & $\begin{array}{c}(0.132307704 \\
0.423029865 \\
0.896404152)\end{array}$ & $\begin{array}{c}(-0.144813144 \\
0.191104314 \\
0.970828664)\end{array}$ & $\begin{array}{c}(-0.079385752 \\
0.343053066 \\
0.935955392)\end{array}$ & Non-Grashof \\
\hline 9 & $\begin{array}{l}(0.112991721, \\
-0.640804412, \\
-0.759343516)\end{array}$ & $\begin{array}{c}(0.132307704 \\
0.423029865 \\
0.896404152)\end{array}$ & $\begin{array}{c}(-0.220517988 \\
0.040888438 \\
0.974525501)\end{array}$ & $\begin{array}{c}(-0.117683260 \\
0.108289377 \\
0.987129202)\end{array}$ & Non-Grashof \\
\hline 10 & $\begin{array}{l}(0.022025725 \\
0.001167093, \\
0.999756723)\end{array}$ & $\begin{array}{c}(-0.064517837 \\
0.361077537 \\
0.930301274)\end{array}$ & $\begin{array}{c}(0.492939483, \\
0.038769499 \\
-0.869199397)\end{array}$ & $\begin{array}{c}(-0.274214436 \\
0.083312874 \\
0.958052926)\end{array}$ & Non-Grashof \\
\hline 11 & $\begin{array}{l}(0.022025725, \\
0.001167093, \\
0.999756723)\end{array}$ & $\begin{array}{c}(-0.064517837 \\
0.361077537 \\
0.930301274)\end{array}$ & $\begin{array}{c}(0.143339851 \\
-0.128057926 \\
0.981353583)\end{array}$ & $\begin{array}{c}(-0.384721164 \\
0.903628911 \\
0.188266879)\end{array}$ & Non-Grashof \\
\hline 12 & $\begin{array}{l}(0.361605187, \\
-0.540091176, \\
-0.759962638)\end{array}$ & $\begin{array}{c}(0.028188073 \\
0.416188589 \\
0.908841290)\end{array}$ & $\begin{array}{c}(-0.047161083 \\
0.048210769 \\
0.997723185)\end{array}$ & $\begin{array}{c}(0.016393930 \\
0.327925973 \\
0.944561165)\end{array}$ & Non-Grashof \\
\hline 13 & $\begin{array}{l}(0.361605187 \\
-0.540091176 \\
-0.759962638)\end{array}$ & $\begin{array}{c}(0.028188073 \\
0.416188589 \\
0.908841290)\end{array}$ & $\begin{array}{c}(-0.362821588 \\
0.133520721 \\
0.922243304)\end{array}$ & $\begin{array}{c}(-0.085555189 \\
0.117641311 \\
0.989363852)\end{array}$ & $\begin{array}{c}\text { Grashof } \\
\text { link2 is rocker }\end{array}$ \\
\hline 14 & $\begin{array}{l}(0.361605187 \\
-0.540091176 \\
-0.759962638)\end{array}$ & $\begin{array}{c}(0.028188073 \\
0.416188589 \\
0.908841290)\end{array}$ & $\begin{array}{c}(-0.524427163 \\
0.693474453 \\
0.494033737)\end{array}$ & $\begin{array}{c}(-0.000146364 \\
0.399049003 \\
0.916929589)\end{array}$ & $\begin{array}{c}\text { Grashof } \\
\text { link2 is rocker }\end{array}$ \\
\hline 15 & $\begin{array}{c}(-0.288452012 \\
0.286378410 \\
-0.913664513)\end{array}$ & $\begin{array}{c}(0.168479974 \\
-0.248307839 \\
0.953917038)\end{array}$ & $\begin{array}{c}(0.729482779 \\
-0.317806582 \\
0.605684614)\end{array}$ & $\begin{array}{l}(0.278254672 \\
-0.253433757 \\
0.926469464)\end{array}$ & Non-Grashof \\
\hline 16 & $\begin{array}{c}(-0.118601752 \\
0.119440205 \\
0.985732044)\end{array}$ & $\begin{array}{c}(-0.216079194 \\
0.573705934 \\
0.790045115)\end{array}$ & $\begin{array}{l}(0.984872274, \\
-0.110632592, \\
0.133368036)\end{array}$ & $\begin{array}{l}(0.198341622, \\
-0.260471416, \\
0.944889011)\end{array}$ & $\begin{array}{c}\text { Grashof } \\
\text { link2 is crank }\end{array}$ \\
\hline
\end{tabular}



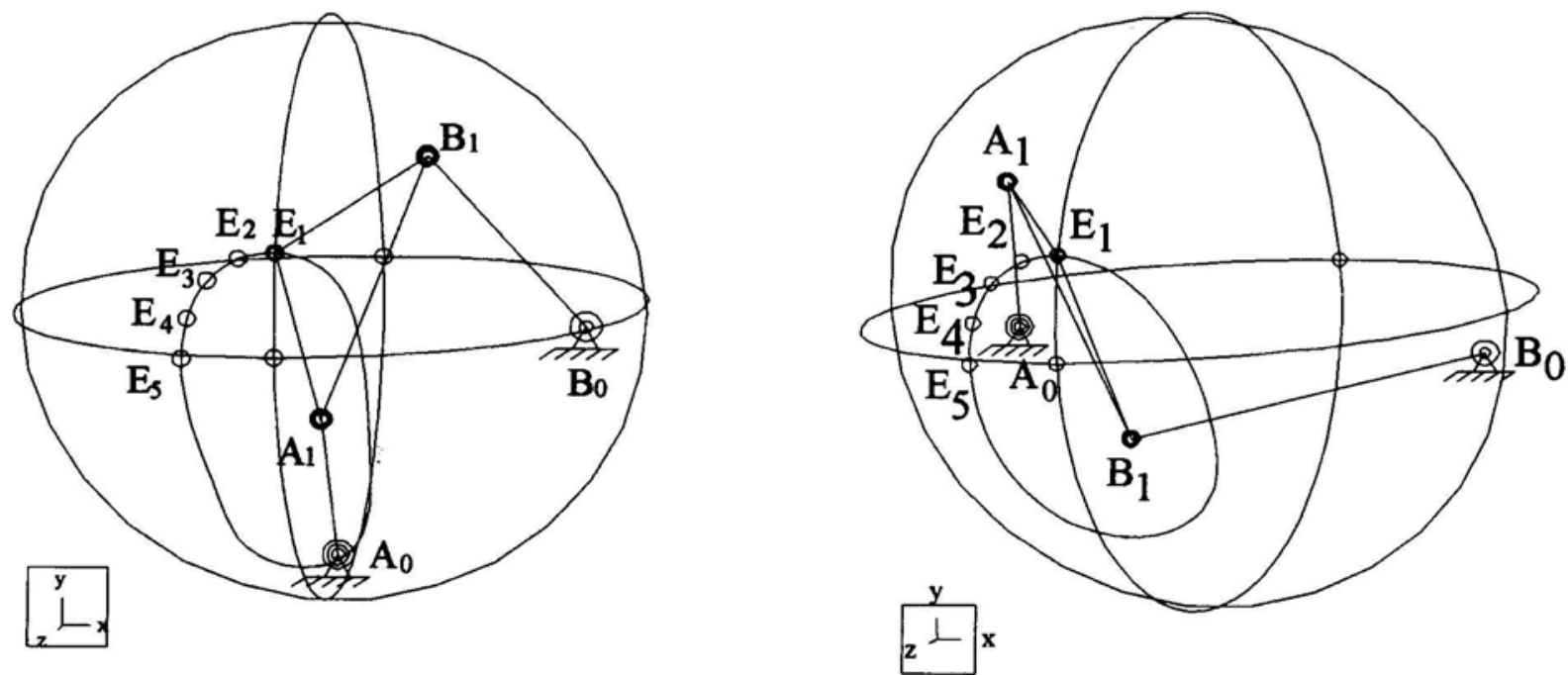

Fig. 4. (A) A Feasible Design of Ex. 1, \#2 in Table 4. (B) A Feasible Design of Ex. 1, \#16 in Table 4.

Table 5. Design specifications of example 2

\begin{tabular}{cccc}
\hline & $\mathrm{x}$ & $\mathrm{y}$ & $\mathrm{z}$ \\
\hline$E_{1}$ & 0.5 & 0.6 & 0.6244998 \\
$E_{2}$ & 0.3 & 0.8 & 0.5196152 \\
$E_{3}$ & -0.1 & 0.8 & 0.5916078 \\
$E_{4}$ & -0.3 & 0.7 & 0.648074 \\
$E_{5}$ & 0.6 & 0.4 & 0.6928203 \\
$A_{0}$ & 0 & 0 & 1 \\
$B_{0}$ & 0.7071067 & 0 & 0.7071067 \\
\hline
\end{tabular}

The solutions in the third row of Table 3 shows that the mechanism is a degenerate one with two of its link length equal to zero. Such solutions should be excluded. After all of the $B_{1}$ and $B_{0}$ corresponding to the ten sets of solutions in Table 2 are found, we further examine the rotatability of the mechanisms. Table 4 shows all of the nondegenerate mechanisms that satisfy the specifications. Among the sixteen mechanisms, only \#1 and \#16 are feasible because the input link can make full rotations. Fig. 4(a) and (4b) show the coupler curves passing the precision points. The figures are results from the motion analysis package MECHANICA. Note that there is no solution identical to the answer in [9]. The reason is that the coordinates $y_{E i}$ of the precision-points in example 1 have been modified so that the length of the position vector is equal to one.

\section{Path Generation with Ground Pivot Specifications}

\section{Example 2}

Table 5 shows the design specifications of such problem. This example is also taken from Chiang [13].

After the coefficients are derived by Mathematica, we apply the 1-homogeneous homotopy method to solve $\sin \beta_{i}$ and $\cos \beta_{i}(i=2,3,4,5)$ from Eqs. (23), (24), (25) and (26), together with the four computability equations Eq. (27). The Bezout number of the system is 1296. (Note that the Bezout number is 589824 if we use the Suh's formulation [7].) There are sixteen sets of real solutions of obtained. The result of $\beta_{i}$ are shown in Table 6 . Then we substitute $\beta_{i}$ into Eqs. (21) and (22) to find coordinates of $A_{0 i}$ and $B_{0 i}$. Finally, by solving two of the Eq. (14) and (15), together with Eq. (1), we obtain the coordinates of the circle-points $A_{1}$ and $B_{1}$. Their coordinates are listed in Table 7.

We examine the rotatability of the mechanisms in Table 7. The Grashof mechanism is denoted by 'G', and non-Grashof one by 'NG' in Table 7. We further check their branch defects and order defect. As shown in Table 7, the number in the comments denotes the passing sequence of the precision points by the coupler-point; Mechanisms \#2 and \#7 have branch and order defects, while \#6, 8, 10, 11, 12, 14 do not have any defects, and the rest have branch defect only. Note the solution \#9 is the same as the answer from Chiang. Figure 5 (resulting from MECHANICA) demonstrates the passing of precision points by the coupler-point curve of mechanism \#6 in Table 7.

The codes was written in FORTRAN, developed and executed on a $486 / 100 \mathrm{Mz}$ PC. The average execution time is, for the first example, $1.1 \mathrm{~s}$ per path; and for the second example, $25 \mathrm{~s}$ per path. The execu- 
Table 6. The real solutions (of $\beta_{i}$ ) by solving Eqs. (23), (24), (25), (26), (27)

\begin{tabular}{ccccr}
\hline & $\beta_{2}$ (deg.) & $\beta_{3}$ & $\beta_{4}$ & \multicolumn{1}{c}{$\beta_{5}$} \\
\hline 1 & -39.946866 & 28.3783920 & -13.9787580 & 15.1230530 \\
2 & 5.0875094 & 27.188004 & 97.088009 & 80.5363490 \\
3 & 148.51028 & 149.02806 & 51.2429350 & 147.9888200 \\
4 & 7.4954342 & 105.78905 & 43.877985 & 76.9989260 \\
5 & 103.82641 & 29.7136910 & 42.6154860 & 72.8595250 \\
6 & -47.445142 & 33.731007 & 38.679016 & 43.7588320 \\
7 & 7.403504 & -46.034393 & -34.617085 & -5.5326016 \\
8 & 22.351743 & -26.381132 & 38.453362 & 40.7150920 \\
9 & 19.037263 & 45.122867 & 57.692804 & 80.4005010 \\
10 & 23.54982 & 34.41971 & -9.83586 & 35.6038290 \\
11 & 69.394068 & 81.627485 & 86.576073 & 92.2211990 \\
12 & -1.9434876 & 91.16204 & 94.3438790 & 95.7001230 \\
13 & 22.313002 & 52.173802 & 66.976176 & 96.5721160 \\
14 & 98.155703 & 22.847757 & 105.81964 & 104.8521700 \\
15 & -43.316542 & -38.454779 & -33.294229 & 81.2922320 \\
16 & -25.509732 & -10.817697 & 43.095799 & 30.4387110 \\
\hline
\end{tabular}

Table 7. The mechanisms fulfilling the design specifications in Ex. 2

\begin{tabular}{cccccccc}
\hline & \multicolumn{1}{c}{$x_{A 1}$} & \multicolumn{1}{c}{$y_{A 1}$} & $z_{A 1}$ & \multicolumn{1}{c}{$x_{B 1}$} & \multicolumn{1}{c}{$y_{B 1}$} & $z_{B 1}$ & Comments \\
\hline 1 & 0.3423927 & -0.1296145 & 0.9305737 & 0.8109208 & -0.3545551 & 0.4655085 & $\begin{array}{c}\text { NonGrashof, } \\
\text { pass 1-2-3 }\end{array}$ \\
2 & -0.6226947 & -0.0167462 & 0.7822856 & 0.18050342 & -0.1203403 & 0.9761848 & NG,1-4-5-3 \\
3 & 0.7292376 & 0.5019565 & 0.4650293 & -0.0870786 & 0.2047293 & 0.9749376 & Grashof, pass 1-4 \\
4 & -0.6523399 & 0.1415521 & 0.7445909 & 0.12665916 & -0.0497913 & 0.9906959 & NG, 1-3 \\
5 & -0.4797264 & 0.4151703 & 0.7729788 & 0.1225811 & -0.0611761 & 0.9905712 & NG,1-3 \\
6 & -0.0923931 & -0.7463575 & 0.6591009 & 0.8169919 & -0.4335955 & 0.3801567 & G,1-2-3-4-5 \\
7 & 0.4497704 & -0.1932066 & 0.8719964 & 0.9373027 & -0.1896528 & 0.2923961 & NG,1-4-5-2 \\
8 & -0.1234608 & -0.8140431 & 0.5675308 & 0.7827079 & -0.4172019 & 0.4618560 & G,1-2-3-4-5 \\
9 & -0.0921916 & 0.0586758 & 0.9940110 & -0.1839569 & 0.3963604 & 0.8994767 & G,1-2-3-4 \\
10 & -0.1671290 & -0.8945945 & 0.4144498 & 0.7574083 & -0.3999633 & 0.5161027 & G,1-2-3-4-5 \\
11 & -0.7137237 & -0.2242567 & 0.6635567 & 0.3090444 & -0.4699837 & 0.8268052 & NG,1-2-3-4-5 \\
12 & -0.8566417 & -0.3517913 & 0.3773697 & 0.2212870 & -0.3009001 & 0.9276266 & G,1-2-3-4-5 \\
13 & -0.0910223 & 0.1360747 & 0.9865083 & -0.0732288 & 0.4223394 & 0.9034750 & NG,1-2-3-4 \\
14 & 0.8803770 & 0.4718230 & 0.0481603 & 0.1317618 & -0.1677727 & 0.9769806 & G,1-2-3-4-5 \\
15 & 0.5005738 & 0.1410012 & 0.8541338 & 0.5515259 & -0.7745495 & 0.3096649 & G,1-2-3-4 \\
16 & 0.2506777 & -0.1163524 & 0.9610530 & 0.6830587 & -0.4107287 & 0.6039311 & G,1-2-3 \\
\hline
\end{tabular}

tion time depends heavily on the tolerance criterion setting. Here we shall not discuss the convergence criterion vs. CPU time.

\section{CONCLUSIONS}

In contrast to local numerical schemes such as Newton's method, continuation method is a globally convergent numerical method that can obtain the complete solution set of a problem. In this paper, we have developed a reliable computer code, and demonstrated the effectiveness of continuation method in solving the synthesis of spherical four-bar linkages, including body-guidance and path generation problems.

In the derivation of the design equations, we use the concept of graphical inversion approach, and the concept of the pole method. We've also modified the procedure of the pole method in order to reduce the system order. The formulation has the advantages in that (1) the design equations are in the form of polynomial system equations, (2) the total degree of the system is less than that of other approaches, (3) it can handle various design specifications. It is shown that, 


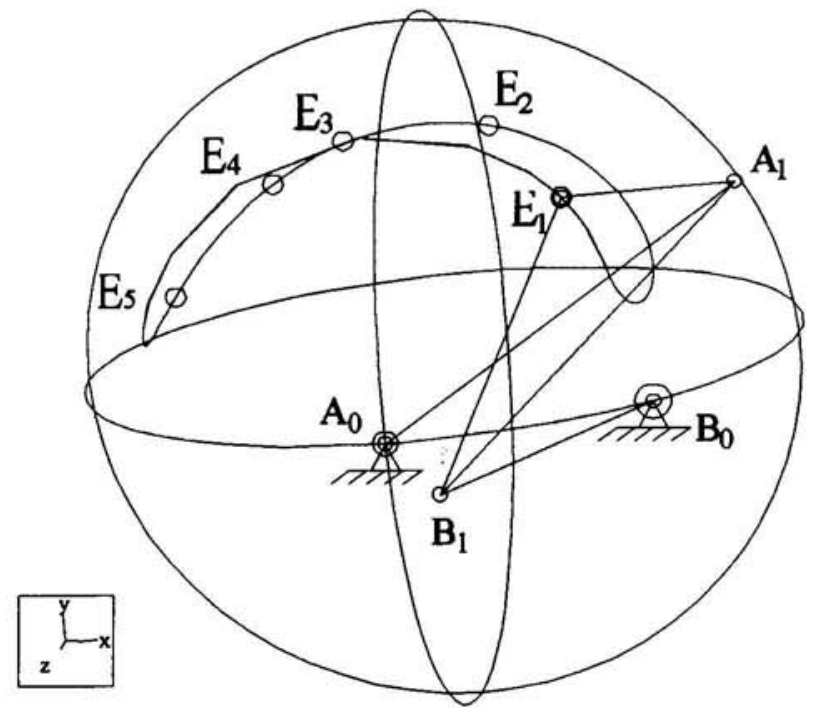

Fig. 5. A Feasible Design of Ex. 2, \#14 in table 7.

for the problems of five-position motion or path generation with prescribed crank angles, the total degree of the system is at most $18 \times 18=324$. Even for the problem of path generation with ground pivot specifications, the Bezout number is 1296 , which is considered a small order. In the two examples, we've successfully compute all of the solutions of each problem. Further implementations is under development for the nine-point synthesis problems.

\section{REFERENCES}

1. Sandor, G.N. and Erdman, A.G., Advanced Mechanism Design: Analysis and Synthesis, Vol. 2, PrenticeHall, Inc., NJ, (1989).

2. Freudenstein, F. and Sandor, G.N., "Synthesis of PathGenerating Mechanisms by Means of a Programmed Digital Computer," ASME Journal of Engineering for Industry, 81B, pp. 159-167 (1959).

3. Lin, C.K. and Chiang, C.H., "Synthesis of Planar and Spherical Geared Five-Bar Function Generators By the Pole Method," Mechanism and Machine Theory, Vol. 27, No. 2, pp. 131-141 (1992).

4. Morgan, A.P. and Wampler, C.W., "Solving Planar Four-Bar Design Problem Using Continuation," ASME Journal of Mechanical Design, Vol. 112, No. 4, pp. 544-550 (1990).

5. Roth, B. and Freudenstein, F., "Synthesis of PathGenerating Mechanisms by Numerical Methods," ASME Journal of Engineering for Industry, Vol. 85B, No. 3, pp. 298-306 (1963).

6. Roth, B., "Finite-Position Theory Applied to Mechanism Synthesis," ASME Journal of Applied Mechanics, Vol. 34, 599-605 (1967).
7. Tsai, L.W. and Lu, J.J., "Coupler-Point-Curve Synthesis Using Homotopy Methods," ASME Journal of Mechanical Design, Vol. 112, No. 3, pp. 384-389 (1990).

8. Tong, S.H. and Chiang, C.H., "Synthesis of Planar and Spherical Four-Bar Path Generators By the Pole Method," Mechanism and Machine Theory, Vol. 27, No. 2, pp. 143-155 (1992).

9. Wampler, C.W. and Morgan, A,P., "Complete Solution of The Nine-Point Path Synthesis Problem for Four-Bar Linkage," Journal of Mechanical Design, pp. 153-159 (1992).

10. Suh, C.H. and Radcliffe, C.W., "Synthesis of Spherical Linkages With Use of The Displacement Matrix," ASME Journal of Engineering for Industry, Vol. 89B, pp. 215-222 (1967).

11. Wampler, C.W., Morgan, A.P. and Sommese, A.J., "Numerical Continuation Methods for Solving Polynomial Systems Arising in Kinematics," ASME Journal of Mechanical Design, Vol. 112, No. 1, pp. 59-68 (1990).

12. Subbian, T. and Flugrad, D.F., "Four-Bar Path Generation Synthesis by Continuation Method," ASME Journal of Mechanical Design, Vol. 113, No. 1, pp. 63-69 (1991).

13. Chiang, C.H., Kinematics of Spherical Mechanisms, Cambridge University Press, NY, (1988).

\section{球面四連桿五個精確位置合成之完

$$
\text { 全解 }
$$

$$
\text { 林鎮 洲 }
$$

國立台灣海洋大學機械與輪機工程學系

$$
\text { 摘 要 }
$$

本文是探討以連續法解球面四連桿之運動產 生機構與路徑產生機構的合成問題。連續法所求得 的解是系統全部的解, 可以提供機構設計者符合運 動規籍之所有機構。此乃基於連續法是一種具整體 收敛性的數值方法, 不同於牛顿法或鲍威爾法等局 部性收敛數值方法数初始猜值非常敏銳, 故連續法 能保證求得全部的解。本文主要針缽五個精確位置 的問題探討, 並且比較過去的結果。設計方程式的 推導是根據倒置法與極心法的觀念而得, 其優點是 設計方程式較簡單, 系統的乘举次数較低。故以連 續法求解時較有效率。根據以上方法發展的電腦程 式將解出全部的合成機構, 最後再根據機構的可轉 動性分類, 並且检驗其迴路缺陷與次序缺陷。 